\title{
An exploratory analysis of common genetic variants in the vitamin D pathway including genome-wide associated variants in relation to glioma risk and outcome
}

\author{
Gabriella M. Anic, \\ Department of Cancer Epidemiology, H. Lee Moffitt Cancer Center \& Research Institute, Tampa, \\ FL 33612, USA \\ Reid C. Thompson, \\ Department of Neurological Surgery, Vanderbilt University Medical Center, Nashville, TN 37232 , \\ USA
}

\section{Burton Nabors,}

Neuro-oncology Program, University of Alabama at Birmingham, Birmingham, AL 35294, USA

Jeffrey J. Olson,

Department of Neurosurgery, Emory School of Medicine, Atlanta, GA 30322, USA

\author{
James E. Browning, \\ Department of Cancer Epidemiology, H. Lee Moffitt Cancer Center \& Research Institute, Tampa, \\ FL 33612, USA
}

\section{Melissa H. Madden,}

Department of Cancer Epidemiology, H. Lee Moffitt Cancer Center \& Research Institute, Tampa, FL 33612, USA

\section{F. Reed Murtagh,}

Department of Diagnostic Imaging, H. Lee Moffitt Cancer Center \& Research Institute, Tampa, FL 33612, USA

Peter A. Forsyth, and

Department of Neuro-Oncology, H. Lee Moffitt Cancer Center and Research Institute, Tampa, FL 33612, USA

\section{Kathleen M. Egan}

Department of Cancer Epidemiology, H. Lee Moffitt Cancer Center \& Research Institute, Tampa, FL 33612, USA

Kathleen M. Egan: kathleen.egan@moffitt.org

\begin{abstract}
Purpose-Experimental and epidemiological evidence shows a beneficial role of vitamin D in cancer. In vitro evidence is consistent with a similar protective function in glioma; however, no study has yet examined the potential role of vitamin $\mathrm{D}$ in glioma.
\end{abstract}

Methods-We evaluated the association between common genetic variants in the vitamin D pathway and glioma risk and patient outcome in 622 newly diagnosed glioma cases and 628

\footnotetext{
(C) Springer Science+Business Media B.V. 2012

Correspondence to: Kathleen M. Egan, kathleen. egan@moffitt.org.

Conflict of interest The authors have no conflicts of interest.
} 
healthy controls enrolled in a clinic-based case-control study. Subjects were genotyped for 7 candidate and tagging single nucleotide polymorphisms in the vitamin $\mathrm{D}$ receptor and 8 additional variants in NADSYN1, GC, CYP24A1, CYP2R1, and C1OORF88 linked in genome-wide association studies to serum concentrations of vitamin D. Unconditional logistic regression was used to estimate age- and gender-adjusted odds ratios and $95 \%$ confidence intervals for glioma risk according to vitamin D genotypes. Proportional hazards regression was used to estimate hazard ratios for glioma-related death among 320 patients diagnosed with high-grade tumors. $P$ values were uncorrected for multiple comparisons.

Results-Risk of astrocytic tumors was associated with variant alleles in rs3829251 (NADSYN1), rs10741657 (CYP2R1), rs2228570 (Fok1, VDR), and rs731236 (Taq1, VDR). No risk associations were found among oligodendroglial tumors. Survival associations were observed according to variant status for rs1544410 (Bsm1, VDR) and rs6013897 (CYP24A1).

Conclusion-This exploratory analysis provides limited evidence of a role for genetic variation in vitamin D pathway genes with glioma risk and survival.

\section{Keywords}

Glioma; Vitamin D; Single nucleotide polymorphism; Genotype; VDR

\section{Introduction}

Gliomas are an aggressive primary tumor of the brain with unknown etiology. The vitamin D metabolite $1,25(\mathrm{OH})_{2} \mathrm{D}_{3}$ may protect against cancer by promoting cell differentiation and apoptosis and by inhibiting cell proliferation and angiogenesis (reviewed in [1]). Findings from epidemiologic studies investigating the association between serum levels of $1,25(\mathrm{OH})_{2} \mathrm{D}_{3}$ and cancer risk have been inconsistent though [2-6]; the only study to consider brain cancer did not observe a significant association between plasma vitamin $\mathrm{D}$ levels and risk of brain tumors of all types [7]. However, the observation that there are higher incidence rates of glioma in regions with lower ambient ultraviolet $\mathrm{B}$ radiation [8], the principal source of vitamin $\mathrm{D}$ in humans, and in vitro evidence that $1,25(\mathrm{OH})_{2} \mathrm{D}_{3}$ inhibits growth and induces apoptosis in glioma cell lines [9] suggests a potential role for vitamin D in glioma.

The effects of $1,25(\mathrm{OH})_{2} \mathrm{D}_{3}$ are mediated through the vitamin $\mathrm{D}$ receptor (VDR), a transcription factor expressed in multiple tissue types including the brain [10]. Single nucleotide polymorphisms (SNPs) in the VDR gene, located on chromosome 12q13, influence the activity of $1,25(\mathrm{OH})_{2} \mathrm{D}_{3}$. The Fok1 (rs2228570) and $C d \times 2$ (rs11568820) polymorphisms alter transcription of the $V D R$ gene [11], while the functional relevance of other commonly studied $V D R$ polymorphisms including Taq1 (rs731236), Apa1 (rs7975232), and Bsm1 (rs1544410) is unclear [12]. No study has yet examined whether genetic variants in VDR or SNPs associated with serum concentrations of 25-

hydroxyvitamin D in genome-wide association (GWA) studies $[13,14]$ are related to glioma risk or patient outcome.

We evaluated these potential associations in a series of 622 newly diagnosed glioma cases and 628 healthy controls enrolled in the Study of Glioma in the Southeast (GliomaSE), a multi-center, clinic-based case-control study conducted at medical centers in the Southeastern United States. 


\section{Subjects and methods}

\section{Study population}

A description of the study population has been published previously $[15,16]$. Briefly, cases were Caucasian individuals aged 18 and older recently diagnosed (within 3 months) with a primary, non-recurrent glioma. Cases were identified at neurosurgery and neuro-oncology clinics at major medical and oncology centers in the Southeastern United States including Vanderbilt University Medical Center in Nashville, Tennessee; Moffitt Cancer Center in Tampa, Florida; the University of Alabama at Birmingham; Emory University in Atlanta, Georgia; and the Kentuckiana Cancer Institute in Louisville, Kentucky. As eligibility in the case-control study required a recent diagnosis of glioma, only primary glioblastoma multiforme (GBM) and de novo anaplastic astrocytoma were included in the case group. Controls included friends and other non-blood-related associates of the cases as well as residents from the same communities as the cases identified in white page listings. Controls were excluded if they reported a personal history of a brain tumor. Eighty-seven percent of eligible glioma patients were enrolled in the study, a median of 1.0 month following the glioma diagnosis (interquartile range: 2 weeks -1.7 months). Study protocols were approved by the institutional review committees at each participating center and all study participants provided written informed consent.

Interviewer-administered questionnaires were used to collect data on demographic characteristics and potential glioma risk factors. Genomic DNA samples were self-collected by oral rinse or the saliva method using Oragene kits (www.dnagenotek.com).

\section{DNA processing and genotyping}

DNA was extracted and stored at the Core Genotyping Facility at Vanderbilt (during the pilot phase) or at the Tissue Core laboratory of Moffitt Cancer Center (the coordinating center). For the present analysis, we examined 8 SNPs in the $V D R$, including the commonly studied restriction fragment length polymorphisms (RFLPs) Apa1, Taq1, Bsm1, Fok1, and $C d \times 2$, and 3 tagging SNPs. An additional 10 SNPs linked to serum concentrations of 25hydroxyvitamin D levels in $G C$ (4q12-q13), CYP2R1 (11p15), CYP24A1 (20q13), NADSYN1 (11q13), and C10orf88 (10q26) [13, 14] were also genotyped. Genotyping was performed at the Center for Genome Technology at the Hussman Institute for Human Genomics, University of Miami using Illumina's GoldenGate technology (Illumina, San Diego, CA). Genotyping by Taqman was carried out for SNPs that failed on the Illumina array. A total of 655 glioma cases and 658 controls, all Caucasian, were submitted for genotyping. Quality control samples (water, CEPH DNA, as well as blinded and unblinded DNA samples) were included in genotyping runs. Laboratory staff was blinded to the casecontrol status of the samples. Two SNPs (VDR rs7975232 (Apa1) and CYP2R1 rs2060793) failed genotyping and in one additional SNP $(G C$ rs 12512631) there was departure from Hardy-Weinberg Equilibrium among the controls ( $p$ value of $<0.01$ ). Concordance of genotype calls in 94 blinded duplicate pairs ranged from 89 to $100 \%$ (mean, $99.6 \%$ ) among the 15 successfully genotyped SNPs. The genotyping success rate for individuals ranged from 91.4 to $99.7 \%$ (mean, $97.6 \%$ ). Glioma risk has also been associated with established susceptibility variants for these tumors $[17,18]$ in this case-control series [15].

\section{Statistical analysis}

Risk associations were modeled using unconditional logistic regression with odds ratios (ORs) and $95 \%$ confidence intervals (CIs) for individual genotypes adjusted for age and gender. To test for linear trend, each SNP was modeled as an ordinal term coded 0,1 , and 2 corresponding to the number of variant alleles. Multinomial logistic regression was used to examine associations between genotypes and histologic subtypes of glioma (GBM, low- 
grade astrocytomas, and oligodendrogliomas). A test for heterogeneity was performed in each multinomial model to evaluate if the odds ratios for each SNP were significantly different across histology groups. Survival associations were examined among the 320 patients with high-grade tumors (GBM, anaplastic astrocytoma, and high-grade oligodendrogliomas) treated with the current standard of care (surgical resection, radiation, and temozolomide) as a first course of therapy. Cox proportional hazards regression was used to evaluate the prognostic influence of vitamin D polymorphisms on glioma survival, adjusting for age, gender, and histologic subtype of glioma (GBM; high-grade astrocytoma (non-GBM); high-grade oligodendroglioma). All $p$ values were two sided and uncorrected for multiple comparisons. Analyses were performed using SAS 9.2 (SAS Institute, Inc., Cary, USA).

\section{Results}

Table 1 presents characteristics of glioma cases and controls. The median age among cases was 52 years (range: 18-88) and among controls was 56 years (range: 18-87). The majority of cases $(63 \%)$ and controls $(57 \%)$ were male. Subjects resided in Tennessee (30\%), Florida (25\%), Alabama (14\%), Kentucky (13\%), Georgia (11\%), and other southern states $(7 \%)$.

Associations with glioma risk under an additive model are shown in Table 2. A positive association was observed with increasing number of variant alleles in NADSYN1 rs3829251 $(\mathrm{OR}=1.26$; $95 \%$ CI: $1.00-1.58 ; p=0.05)$; no other significant associations were evident. We further examined associations under dominant and recessive models (data not shown) and found an inverse association under a dominant model for VDR Fok 1 (OR $=0.77 ; 95 \%$ CI: 0.61-0.98; $p=0.03$ ) and $C Y P 2 R 1$ rs 10741657 (OR $=0.72 ; 95 \%$ CI: 0.56-0.92; $p=$ $0.01)$.

Risk associations according to histologic subtype of glioma are shown in Table 3 . The positive association with $N A D S Y N 1$ rs3829251 was restricted to high-grade (GBM) and lower-grade astrocytic tumors (combined OR $=1.32 ; 95 \%$ CI: $1.04-1.67 ; p=0.02$ ) with no similar association in oligodendrogliomas ( $\mathrm{OR}=0.89 ; 95 \%$ CI: $0.55-1.46 ; p=0.65)$ ( $\mathrm{p}$ for heterogeneity $=0.18$ ). There was some evidence of heterogeneity across histology types for CYP2R1 rs10741657; an inverse association was observed in GBM and lower-grade astrocytic tumors combined $(\mathrm{OR}=0.79 ; 95 \% \mathrm{CI}: 0.65-0.95 ; p=0.01)$ but not in oligodendrogliomas $(\mathrm{OR}=1.17 ; 95 \% \mathrm{CI}: 0.82-1.68 ; p=0.37)(\mathrm{p}$ for heterogeneity $=0.08)$. Homozygous variant carriers of the VDR Taq 1 variant were at significantly reduced risk of GBM only (OR $=0.58 ; 95 \%$ CI: 0.35 to $0.94 ; p=0.03$ ) (data not shown).

We examined genotype associations with glioma survival in 320 patients with high-grade tumors treated with the current standard of care (surgical resection, radiation, and temozolomide) (Table 4). A total of 248 deaths from glioma were documented (median follow up among 72 surviving patients: 28 months). Reduced survival was observed among variant allele carriers in $V D R B s m 1$ (HR $=1.34 ; 95 \%$ CI: $1.01-1.77 ; p=0.04)$ under a dominant model. Variant carriers of $C Y P 24 A 1 \mathrm{rs6013897}$ had significantly prolonged survival under both additive (per allele $\mathrm{HR}=0.79 ; 95 \% \mathrm{CI}$ : 0.63-0.98; $\mathrm{p}$ for trend $=0.03$ ) and recessive $(\mathrm{HR}=0.54 ; 95 \% \mathrm{CI}: 0.30-0.96 ; p=0.04)$ models.

We attempted to validate survival associations in the Cancer Genome Atlas (TCGA) (downloaded from http://tcga-data.nci.nih.gov/tcga) [19] among 211 TCGA GBM patients that underwent surgery, radiation, and chemotherapy. Neither of the 2 variants associated with survival in the current series (rs1544410 (Bsm1) and rs17217119 as a proxy for CYP24A1 rs6013897 $\left(I^{2}=1.0\right)$ ) was associated with glioma survival in TCGA (not shown). 


\section{Discussion}

To our knowledge, this is the first evaluation of common polymorphisms in the vitamin D pathway in relation to glioma. In this large series of incident glioma cases, suggestive associations were identified for SNPs in the VDR and in genes NADSYN1, CYP2R1, and $C Y P 24 A 1$. Risk associations were observed primarily for astrocytic tumors whereas no significant associations were evident for oligodendrogliomas. Associations with survival were also suggested for two of the examined variants, although neither finding was validated in the Cancer Genome Atlas data.

RFLPs Bsm1 and Taq1, which are in high linkage disequilibrium in the $3^{\prime}$ untranslated region of the $V D R$ [12], are among the $V D R$ SNPs most commonly studied in relation to cancer risk. Though Taq1 and $B s m 1$ have no known functional relevance and do not change the structure of the VDR protein, they are strongly linked to a poly(A) microsatellite repeat in the $3^{\prime}$ untranslated region that may be associated with VDR mRNA stability [20]. Our findings suggesting a reduced risk for astrocytic tumors associated with the Taq1 variant are consistent with findings indicating a similar pattern for melanoma [21], although no consistent association has emerged with this variant and other malignancies (reviewed in [22, 23]). The association between $B s m 1$ and other cancer types is also inconclusive [22, 23]. Among studies of cancer outcome, a pooled analysis of prospective studies indicated that the $B s m 1$ variant was protective for the development of more aggressive forms of breast cancer [24] whereas in the current study of glioma Bsm 1 carrier status was associated with more aggressive tumors and a worse survival outcome.

The VDR Fok 1 SNP is functionally relevant; the variant $f(\mathrm{~T})$ allele in Fok 1 produces a VDR protein that is three amino acids longer than the wild-type $F(\mathrm{C})$ allele and is functionally less effective [11]. The association between Fok 1 and cancer risk is unclear [21-23]. In the present data, we observed a reduced glioma risk associated with the Fok1 variant under a dominant model across all glioma subtypes.

Several of the SNPs associated with glioma risk or outcome in these analyses emerged in GWA studies as predictors of serum 25-hydroxyvitamin D [13, 14]. The $C Y P 2 R 1$ gene encodes a cytochrome $\mathrm{P} 450$ enzyme that catalyzes the hydroxylation of vitamin $\mathrm{D}$ to the active form of vitamin D. The variant allele in $C Y P 2 R 1$ rs 10741657 has been associated with higher vitamin $D$ levels in several studies $[13,25,26]$ and was associated with reduced glioma risk in the present data. Glioma cases that were carriers of the CYP24A1 rs6013897 variant, associated with lower serum vitamin D [13], had improved glioma survival.

Strengths of this study include the relatively large sample of cases given the rarity of glioma, rapid ascertainment of cases minimizing the potential for survival bias, and the high quality of genotype data, as evidenced by the demonstration of risk associations with established susceptibility alleles in this series of cases and controls [15]. A limitation is that we were not able to examine several variants (GC rs12512631 and CYP2R1 rs2060793) linked to serum vitamin D levels $[10,11]$ that failed genotyping in the present study. While the study implicated several functional variants in the VDR and provided suggestive findings for variants linked to vitamin $\mathrm{D}$ status, many associations were examined in this exploratory analysis and $p$ values were uncorrected for multiple comparisons; therefore, significant findings may have arisen by chance. Population stratification may also have influenced our findings and results from this exploratory analysis need to be replicated in further studies.

In summary, this exploratory analysis provides some limited evidence supporting a role for vitamin D in glioma onset and survival of glioma. 


\section{Acknowledgments}

The authors wish to acknowledge study participants for their contributions. We further wish to thank the clinicians and research staffs at participating medical centers. Special thanks go to Celia Sigua and Marek Wloch of the Tissue Core at Moffitt and Cara Sutcliffe of the Center for Human Genetics Research DNA Resources Core at Vanderbilt University School of Medicine for their work in processing DNA samples, and Ms. Anna Konidari and staff at the Center for Genome Technology at the Hussman Institute for Human Genomics, University of Miami for their expert technical assistance in the genotyping phase of the study. This study was supported by a grant from the National Institutes of Health (CA R01CA116174), a cancer prevention fellowship for Gabriella Anic supported by the National Cancer Institute grant R25T CA147832, as well as institutional funding from the Moffitt Cancer Center, Tampa, FL, and the Vanderbilt-Ingram Comprehensive Cancer Center, Nashville, TN.

\section{References}

1. Hansen CM, Binderup L, Hamberg KJ, Carlberg C. Vitamin D and cancer: effects of 1,25(OH)2D3 and its analogs on growth control and tumorigenesis. Front Biosci. 2001; 6:D820-D848. [PubMed: 11438443]

2. Garland CF, Gorham ED, Mohr SB, et al. Vitamin D and prevention of breast cancer: pooled analysis. J Steroid Biochem Mol Biol. 2007; 103:708-711. [PubMed: 17368188]

3. Touvier M, Chan DS, Lau R, et al. Meta-analyses of vitamin d intake, 25-hydroxyvitamin d status, vitamin d receptor polymorphisms, and colorectal cancer risk. Cancer Epidemiol Biomarkers Prev. 2011; 20:1003-1016. [PubMed: 21378269]

4. Freedman DM, Looker AC, Chang SC, Graubard BI. Prospective study of serum vitamin D and cancer mortality in the United States. J Natl Cancer Inst. 2007; 99:1594-1602. [PubMed: 17971526]

5. Giovannucci E, Vitamin D. and cancer incidence in the Harvard cohorts. Ann Epidemiol. 2009; 19:84-88. [PubMed: 18291673]

6. Helzlsouer KJ. Overview of the cohort consortium Vitamin D pooling project of rarer cancers. Am J Epidemiol. 2010; 172:4-9. [PubMed: 20562193]

7. Giovannucci E, Liu Y, Rimm EB, et al. Prospective study of predictors of vitamin D status and cancer incidence and mortality in men. J Natl Cancer Inst. 2006; 98:451-459. [PubMed: 16595781]

8. Mohr SB, Gorham ED, Garland CF, Grant WB, Garland FC. Low ultraviolet B and increased risk of brain cancer: an ecological study of 175 countries. Neuroepidemiology. 2010; 35:281-290. [PubMed: 20948235]

9. Elias J, Marian B, Edling C, et al. Induction of apoptosis by vitamin D metabolites and analogs in a glioma cell line. Recent Results Cancer Res. 2003; 164:319-332. [PubMed: 12899531]

10. Eyles DW, Smith S, Kinobe R, Hewison M, McGrath JJ. Distribution of the vitamin D receptor and 1 alpha-hydroxylase in human brain. J Chem Neuroanat. 2005; 29:21-30. [PubMed: 15589699]

11. Uitterlinden AG, Fang Y, Van Meurs JB, Pols HA, Van Leeuwen JP. Genetics and biology of vitamin D receptor polymorphisms. Gene. 2004; 338:143-156. [PubMed: 15315818]

12. Fang Y, van Meurs JB, d'Alesio A, et al. Promoter and 3 '-untranslated-region haplotypes in the vitamin d receptor gene predispose to osteoporotic fracture: the Rotterdam study. Am J Hum Genet. 2005; 77:807-823. [PubMed: 16252240]

13. Wang TJ, Zhang F, Richards JB, et al. Common genetic determinants of vitamin D insufficiency: a genome-wide association study. Lancet. 2010; 376:180-188. [PubMed: 20541252]

14. Ahn J, Yu K, Stolzenberg-Solomon R, et al. Genome-wide association study of circulating Vitamin D levels. Hum Mol Genet. 2010; 19:2739-2745. [PubMed: 20418485]

15. Egan KM, Thompson RC, Nabors LB, et al. Cancer susceptibility variants and the risk of adult glioma in a US case-control study. J Neurooncol. 2011

16. Permuth-Wey J, Thompson RC, Burton Nabors L, et al. A functional polymorphism in the premiR-146a gene is associated with risk and prognosis in adult glioma. J Neurooncol. 2011; 105:639-646. [PubMed: 21744077]

17. Shete S, Hosking FJ, Robertson LB, et al. Genome-wide association study identifies five susceptibility loci for glioma. Nat Genet. 2009; 41:899-904. [PubMed: 19578367] 
18. Wrensch M, Jenkins RB, Chang JS, et al. Variants in the CDKN2B and RTEL1 regions are associated with high-grade glioma susceptibility. Nat Genet. 2009; 41:905-908. [PubMed: 19578366]

19. Cancer Genome Atlas Research Network. Comprehensive genomic characterization defines human glioblastoma genes and core pathways. Nature. 2008; 455:1061-1068. [PubMed: 18772890]

20. Whitfield GK, Remus LS, Jurutka PW, et al. Functionally relevant polymorphisms in the human nuclear Vitamin D receptor gene. Mol Cell Endocrinol. 2001; 177:145-159. [PubMed: 11377830]

21. Denzer N, Vogt T, Reichrath J. Vitamin D receptor (VDR) polymorphisms and skin cancer: a systematic review. Dermatoendocrinol. 2011; 3:205-210. [PubMed: 22110781]

22. Kostner K, Denzer N, Muller CS, Klein R, Tilgen W, Reichrath J. The relevance of vitamin D receptor (VDR) gene polymorphisms for cancer: a review of the literature. Anticancer Res. 2009; 29:3511-3536. [PubMed: 19667145]

23. McCullough ML, Bostick RM, Mayo TL. Vitamin D gene pathway polymorphisms and risk of colorectal, breast, and prostate cancer. Annu Rev Nutr. 2009; 29:111-132. [PubMed: 19400699]

24. McKay JD, McCullough ML, Ziegler RG, et al. Vitamin D receptor polymorphisms and breast cancer risk: results from the National Cancer Institute Breast and Prostate Cancer Cohort Consortium. Cancer Epidemiol Biomarkers Prev. 2009; 18:297-305. [PubMed: 19124512]

25. Davies JR, Chang YM, Snowden H, et al. The determinants of serum vitamin D levels in participants in a melanoma case-control study living in a temperate climate. Cancer Causes Control. 2011; 22:1471-1482. [PubMed: 21853245]

26. Simon KC, Munger KL, Kraft P, Hunter DJ, De Jager PL, Ascherio A. Genetic predictors of 25hydroxyvitamin D levels and risk of multiple sclerosis. J Neurol. 2011; 258:1676-1682. [PubMed: 21431378]

27. Louis DN, Ohgaki H, Wiestler OD, et al. The 2007 WHO classification of tumours of the central nervous system. Acta Neuropathol. 2007; 114:97-109. [PubMed: 17618441] 


\section{Table 1}

Selected characteristics of glioma cases and controls

\begin{tabular}{lcc}
\hline & Cases $(N=\mathbf{6 2 2})$ & Controls $(N=\mathbf{6 2 8})$ \\
\hline Age, median (range) & $52(18-88)$ & $56(18-87)$ \\
Gender, $N(\%)$ & $389(63)$ & $360(57)$ \\
$\quad$ Male & $233(37)$ & $268(43)$ \\
Female & & \\
State of residence, $N(\%)$ & $163(26)$ & $207(33)$ \\
TN & $168(27)$ & $149(24)$ \\
FL & $97(16)$ & $80(13)$ \\
AL & $83(13)$ & $82(13)$ \\
KY & $72(12)$ & $67(11)$ \\
GA & $39(6)$ & $43(7)$ \\
Other & & - \\
Histology type, $N(\%)$ & $341(55)$ & - \\
Glioblastomas & $146(23)$ & - \\
Lower-grade astrocytomas & $94(15)$ & - \\
Oligodendroglial tumors & $41(7)$ & - \\
Other gliomas & & \\
\hline
\end{tabular}

Unequal column totals indicate missing data 


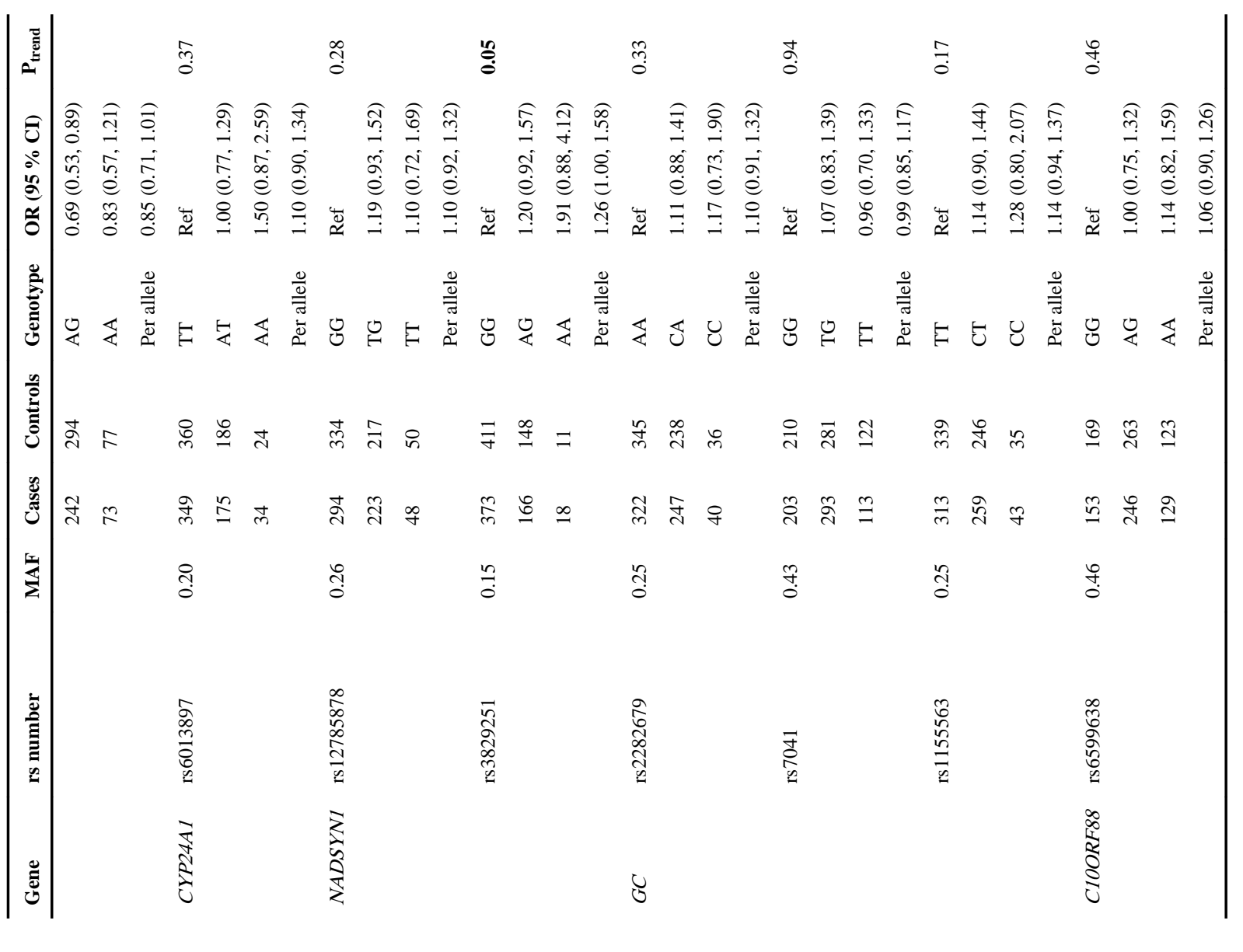




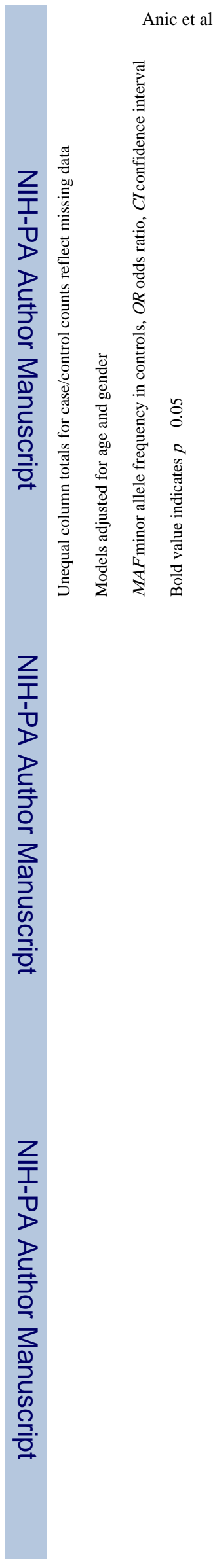

Page 11

Cancer Causes Control. Author manuscript; available in PMC 2013 September 01. 


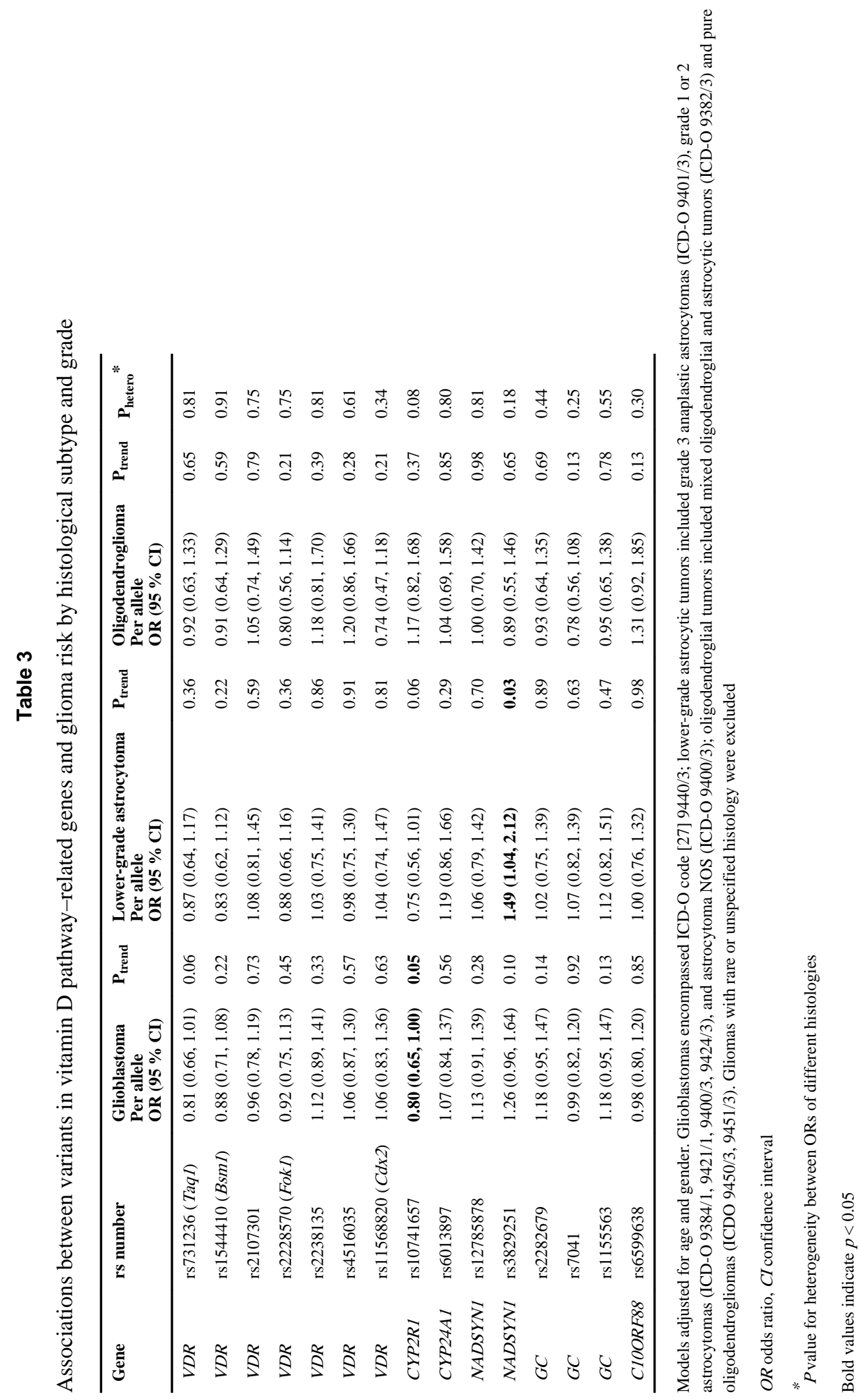


